\title{
JOHN VON NEUMANN AND THE NATIONAL ACCOUNTING MACHINE*
}

\author{
JOHN TODD $\dagger$
}

\begin{abstract}
A visit to D. H. Sadler, Superintendent, H.M. Nautical Almanac Office in 1943 by von Neumann and the author led to one of von Neumann's early contacts with programming.
\end{abstract}

1. Introduction. Early in 1943, von Neumann, who was then interested in explosion problems on behalf of the U.S. Navy, visited the UK. At that time I was organizing an "Admiralty Computing Service" to centralize (where appropriate) mathematical and computational problems arising in Admiralty Establishments and was handling them by way of consultants for the mathematical problems and transferring the computational problems to H.M. Nautical Almanac Office, to which staff had been added for the purpose. The NAO, of which D. H. Sadler was then Superintendent, had a long experience in computational matters and was able to train staff quickly: among those who got experience there were L. Fox, E. T. Goodwin, F. W. J. Oliver and H. H. Robertson.

Although von Neumann was at that time concerned with the formulation and analysis of the gas dynamical problems, he realized that before long, computation would have to be discussed ${ }^{1}$. Accordingly he accompanied me on a visit to the NAO, then evacuated to Bath from Greenwich at the end of April 1943.

The equipment at Bath consisted of Brunsvigas, Marchants and a National Cash Register Accounting Machine, Model 3000. The latter machine had been exploited for scientific purposes by L. J. Comrie (a former Superintendent of the NAO), and some account of this is available in his paper cited below [1].

These applications were mainly for "differencing" or "summation", e.g., building up a table from known sixth differences. Comrie writes that differences up to the fifth can be formed and printed at a rate of some 200 to 300 an hour. These problems only involved the addition or subtraction of the actual entries operations for which the machine was designed.

* Received by the editors September 17, 1973.

$\dagger$ Department of Mathematics, California Institute of Technology, Pasadena, California 91109. This work was supported in part by the National Science Foundation.

${ }^{1}$ I quote from a letter from von Neumann to the author, dated 17 November, 1947.

"I need not tell you that I am exceedingly happy to learn that both Mrs. Todd and you are again in this country and that there is a chance of renewing our pleasant meetings of 1943. It is not necessary for me to tell you what they meant to me and that in particular, I received in that period a decisive impulse which determined my interest in computing machines."

In a paper entitled, Proposal and analysis of a numerical method for the treatment of hydrodynamical shock problems, dated 1944, which is published as no. 95 in vol. 6 of the Collected Works cited below [2], von Neumann discusses some punched card calculations. He also wrote:

"In truly many-dimensional cases the possibility of using other types of machines will also have to be investigated. In this respect the relay-selector type machines seem very promising among the 'digital' ones". 
During our visit to Bath, Mr. Sadler raised the question of adapting the NCR machine to interpolation to halves using the formula

$$
f(3 / 2)=[-f(0)+9 f(1)+9 f(2)-f(3)] / 16,
$$

which is exact if $f$ is a cubic; the error is actually $-\frac{3}{128} h^{4} f^{(4)}(\xi)$ if $f$ has a continuous fourth derivative. It is this problem that we shall discuss.

2. The program. Roughly speaking, the machine with which we were concerned had 6 registers and a keyboard. The operations available are:

entering a number on the keyboard;

printing the contents of a register;

totaling, i.e., adding the contents of a register to others, and clearing the first register;

subtotaling, i.e., adding the contents of a register to others, leaving the first register unchanged.

Direct subtraction, by adding the negative of the contents of a register, is only possible on two registers. $^{2}$

What is required is the following: to be able to enter $f(n)$, carry out a sequence of operations, leading to a printout of

$$
f(n-3 / 2)=[-f(n-3)+9 f(n-2)+9 f(n-1)-f(n)] / 16 .
$$

It will be acceptable if we enter $f(n) / 16$ instead of $f(n)$.

We can interpret this in matrix vector notation as follows: we can think of the contents of the 6 registers as the components of a vector and the operations can be thought of as a matrix multiplication of this vector. Actually we shall only require 5 registers, and in our description we shall make this assumption. The coefficients can be taken as arbitrary integers $\alpha, \beta, \gamma, \delta$ instead of the special ones $-1,9,9,-1$ of (1).

We consider a general step when the registers read

$v^{\prime}(\alpha f(0)+\beta f(1)+\gamma f(2)+\delta f(3), \alpha f(1)+\beta f(2)+\gamma f(3), \alpha f(2)+\beta f(3), \alpha f(3), f(4))$.

If we premultiply this by

$$
A=\left[\begin{array}{lllll}
0 & 1 & 0 & 0 & \delta \\
0 & 0 & 1 & 0 & \gamma \\
0 & 0 & 0 & 1 & \beta \\
0 & 0 & 0 & 0 & \alpha \\
0 & 0 & 0 & 0 & 0
\end{array}\right],
$$

we get

$$
\begin{aligned}
& (A v)^{\prime}=(\alpha f(1)+\beta f(2)+\gamma f(3)+\delta f(4), \\
& \quad \alpha f(2)+\beta f(3)+\gamma f(4), \alpha f(3)+\beta f(4), \alpha f(4), 0) .
\end{aligned}
$$

${ }^{2}$ In fact it is possible to subtract the contents of any of the other registers from the two special registers. We have, however, kept to the original development, which was based on this mistaken impression, rather than make the minor changes in the "program" displayed below. 
If we now enter $f(5)$ in the last component, we will have completed an inductive step, advancing the indices in (1) by unity.

We now have to represent the transformation $A$ as a composition of feasible machine operations. For simplicity we return to the specific $-1,9,9,-1$ case (Table 1).

TABLE 1

Contents of (five) registers

\begin{tabular}{c|c|c|c|c|c|c}
\hline Operation & Stop & Register 1 & Register 2 & Register 3 & Register 4 & Register 5 \\
\hline & & $-f(0)+9 f(1)+9 f(2)-f(3)$ & $-f(1)+9 f(2)+9 f(3)$ & $-f(2)+9 f(3)$ & $-f(3)$ & $f(4)$ \\
T 1 & & 0 & $-f(1)+9 f(2)+9 f(3)$ & $-f(2)+9 f(3)$ & $-f(3)$ & $f(4)$ \\
T 2 & 1 & $-f(1)+9 f(2)+9 f(3)$ & 0 & $-f(2)+9 f(3)$ & $-f(3)$ & $f(4)$ \\
T 3 & 2 & $-f(1)+9 f(2)+9 f(3)$ & $-f(2)+9 f(3)$ & 0 & $-f(3)$ & $f(4)$ \\
T 4 & 3 & $-f(1)+9 f(2)+9 f(3)$ & $-f(2)+9 f(3)$ & $-f(3)$ & 0 & $f(4)$ \\
ST-5 & 4 & $-f(1)+9 f(2)+9 f(3)$ & $-f(2)+9 f(3)$ & $-f(3)$ & $-f(4)$ & $f(4)$ \\
ST-4 & 1 & $-f(1)+9 f(2)+9 f(3)-f(4)$ & $-f(2)+9 f(3)$ & $-f(3)$ & $-f(4)$ & $f(4)$ \\
ST-4 & 5 & $-f(1)+9 f(2)+9 f(3)-f(4)$ & $-f(2)+9 f(3)$ & $-f(3)$ & $-f(4)$ & $2 f(4)$ \\
ST-4 & 5 & $-f(1)+9 f(2)+9 f(3)-f(4)$ & $-f(2)+9 f(3)$ & $-f(3)$ & $-f(4)$ & $3 f(4)$ \\
ST 5 & 2,3 & $-f(1)+9 f(2)+9 f(3)-f(4)$ & $-f(2)+9 f(3)+3 f(4)$ & $-f(3)+3 f(4)$ & $-f(4)$ & $3 f(4)$ \\
ST 5 & 2,3 & $-f(1)+9 f(2)+9 f(3)-f(4)$ & $-f(2)+9 f(3)+6 f(4)$ & $-f(3)+6 f(4)$ & $-f(4)$ & $3 f(4)$ \\
T 5 & 2,3 & $-f(1)+9 f(2)+9 f(3)-f(4)$ & $-f(2)+9 f(3)+9 f(4)$ & $-f(3)+9 f(4)$ & $-f(4)$ & 0 \\
\hline
\end{tabular}

In the first column of Table 1 we have indicated the "operation": the operator has to choose the operation and the operand and punch the corresponding keys. In the second column we have indicated the "stop", the registers into which the operand is added or subtracted. The "stops" are a primitive form of microprogramming and are fastened to a bar, and tabulation from stop to stop progresses like tabulation on an ordinary typewriter. At the end of the cycle, a return stop organizes a carriage return and paper advance. If we enter $f(5)$ in register 5 we are ready to continue the process.

3. Discussion. This program was conceived in the $6: 44$ train from Bath to London probably on 30 April, and I made a fair copy from our notes and included them in letters to Mrs. Todd (from which the above was copied). Mr. Sadler acknowledged this solution of his problem in a letter to me dated 3 May and presented more professional versions - using more complicated stops and the free register. Such a program would have been about twice as long as a fifth differencing program, so that interpolation at the rate of about 100 /hour was possible.

This was, presumably one of the first computer programs with which von Neumann ${ }^{3}$ was concerned. It was a fixed program and involved a lot of human

${ }^{3}$ An early account of von Neumann's considerations of computing machines is a previously unpublished paper (with H. H. Goldstine) dated 1946 which appears as no. 104, vol. 5 of the Collected Works [2]. The more familiar early material are the four reports for U.S. Army Ord. Dept. (written with A. W. Burks and H. H. Goldstine) and dated 1946/8 (reprinted as no. 102, 107, 112, 113 in vol. 5) and the report for the Navy Bur. Ord. (written with V. Bargmann and D. Montgomery) dated 1946 (reprinted as no. 101 in vol. 5).

I am indebted to Professor Brian Randell for pointing out that von Neumann's First draft of a report on the EDVAC, Moore School, University of Pennsylvania, dated 30 June 1945, antedates no. 104. See also D. E. Knuth [4]. 
intervention. The Admiralty Computing Service, which designed and had constructed two special purpose computers, ${ }^{4}$ had contemplated a mechanization of the above program. Using "stops" on a (circular) "form bar" to actuate the control keys (electromechanically) would reduce the manual work to the setting of numbers and the initiation of the cycle.

Actually, no further work was done in connection with these problems.

4. The manuscript. We give below (Figs. 1 and 2) a reproduction of some of the rough work of von Neumann. We recall that this was done in a blacked-out train, on the rather poor quality paper issued to government scientists at that time.

All the relevant papers have been transferred to the Computer History Project, Smithsonian Museum, Washington, D.C.

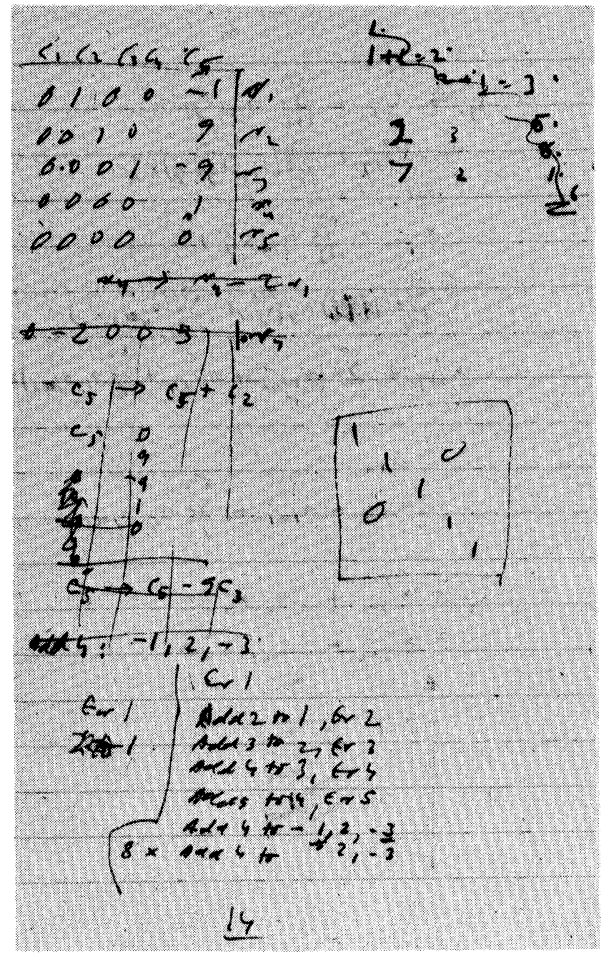

FIG. 1

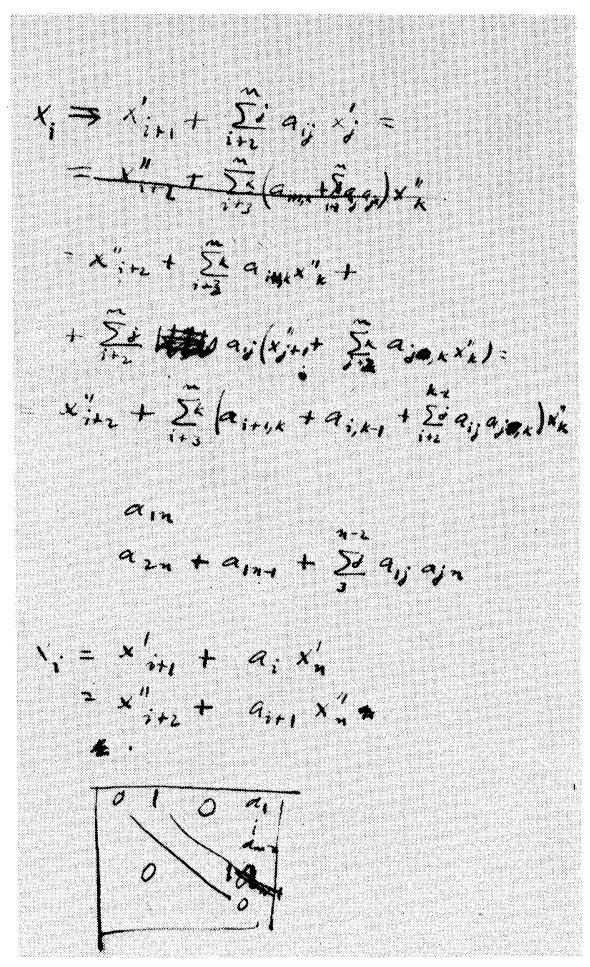

FIG. 2

Acknowledgment. This account is based on notes taken at the time and letters describing them written to Mrs. Todd (Olga Taussky) and on related correspondence with D. H. Sadler. I am indebted to Mr. Sadler for a critical reading of a draft of this paper.

${ }^{4}$ See items 40, 112 in the paper of D. H. Sadler and the author cited below [3]. These activities were largely the responsibility of the late Alan Baxter. 


\section{REFERENCES}

[1] L. J. COMrIE, Inverse interpolation and scientific applications of the National Accounting Machine, J. Royal Statist. Soc. Suppl., 3 (1936), pp. 87-114.

[2] John von Neumann, Collected Works, 6 vols., A. H. Taub, ed., Macmillan, New York, 1961-1963.

[3] D. H. Sadler And J. Todd, Admiralty Computing Service, Math. Tables Aids Computation, 2 $(1966 / 7)$, pp. 289-297.

[4] D. E. KNUTH, Von Neumann's first computer program, Comput. Surveys, 2 (1970), pp. 247--260. 